\title{
Understanding COVID-19 vaccine hesitancy and resistance: another challenge in cancer patients
}

\author{
Nesrine Mejri ${ }^{1}\left[\right.$ ] Yosra Berrazega $^{1} \cdot$ Emna Ouertani ${ }^{1} \cdot$ Haifa Rachdi ${ }^{1} \cdot$ Mariem Bohli ${ }^{2} \cdot$ Lotfi Kochbati $^{2}$. \\ Hamouda Boussen ${ }^{1,3}$
}

Received: 21 May 2021 / Accepted: 5 July 2021 / Published online: 19 July 2021

(c) The Author(s), under exclusive licence to Springer-Verlag GmbH Germany, part of Springer Nature 2021

\begin{abstract}
Introduction We aimed to measure the acceptability towards the COVID-19 vaccination in cancer patients and to investigate determinant factors associated with the patient's choice.

Methods We conducted a cross-sectional survey with a self-administered questionnaire delivered to 329 cancer patients in 3 oncology cancer centers in Tunisia between February-May 2021. Logistic regression was used to evaluate odds ratio predicting patient's intentions toward the vaccine.

Results Acceptance rate was 50.5\%, 28.3\% ( $n=93)$ reported to definitely refuse the vaccine and $21.2 \%(n=70)$ did not make their decision yet. High educational level, history of comorbidities, history of influenza vaccination in the current season, and patient's opinion about the severity of COVID-19 did not predict vaccine resistance. However, patients who think that the vaccine may interfere with treatment efficacy $(\mathrm{OR}=7.28,95 \% \mathrm{CI}[2.5-12.32])$, or may impact cancer outcome $(\mathrm{OR}=6.14$, 95\% CI [2.27-16.7]), were significantly more likely to refuse the vaccine. Patients who disagree that the vaccine is a major weapon against the pandemic $(\mathrm{OR}=6.07,95 \% \mathrm{CI}[2.34-9.52])$ or that it could reduce the virus transmission $(\mathrm{OR}=7.34$, 95\% CI [4.22-11.81]) were also significantly more likely to reject the vaccination. Safety concerns were also significant predictive factors $(\mathrm{OR}=7.9,95 \% \mathrm{CI}$ [4.10-11.27]. Confidence level in the authorities played a significant role in patient's acceptance of the vaccine, indeed patients who are not registered $(\mathrm{OR}=5.9,95 \% \mathrm{CI}$ [1.58-8.7]) or not informed about the Tunisian national vaccination platform $\operatorname{EVAX}(\mathrm{OR}=5.51,95 \% \mathrm{CI}[2.1-7.9])$ were more likely to be against the vaccine.

Conclusion Cancer patient's education about the impact of the vaccine on their disease and on the COVID-19 is needed. Governments should build strategies to gain more population confidence.
\end{abstract}

Keywords COVID-19 $\cdot$ Cancer $\cdot$ Vaccination $\cdot$ Hesitancy

\section{Introduction}

Coronavirus disease 2019 (COVID-19) pandemic impacted cancer patients in several ways leading to late diagnosis, limited access to required health care and impaired management [1]. Furthermore, patients with cancer were considered

Nesrine Mejri

nesrinemejriturki2@gmail.com

1 Medical Oncology Department, Abderrahmane Mami Hospital, Faculty of Medicine, University Tunis El Manar, Tunis, Tunisia

2 Radiation Therapy Department, Abderahmen Mami Hospital, Faculty of Medicine, University Tunis El Manar, Tunis, Tunisia

3 Medical Oncology, Clinic Taoufik, Tunis, Tunisia as a high-risk population with an increased risk of severe infections and an $\sim 3.5$-fold increase in the risk of needing mechanical ventilation or intensive care unit admission/ death compared with patients without cancer [2, 3]. Currently, some advances toward a specific treatment have been made [4] but vaccination is considered to be the most awaiting intervention to stop the virus spread. Previous studies showed that patients with an active cancer should be considered for priority access to COVID-19 vaccination, along other particularly vulnerable populations with risk factors [5]. The Advisory Committee on Immunization Practices within the Centers for Disease Control and Prevention in the USA considered that patients with cancer should be one of the groups considered for early COVID-19 vaccination [6]. Limited supply of the vaccine is one of the major obstacles, but vaccine acceptancy is also considered 
by the Word Health Organization as one the top ten global health threats: high levels of hesitancy lead to low vaccine demand [7]. Vaccine acceptancy is context-specific and varies with geography, culture, and sociodemographic factors. It depends on public trust and confidence in the safety and efficacy of vaccines, the healthcare system, and professionals. Vaccine hesitancy and misinformation could put public health, for instance cancer patients, at risk in responding to the current crisis. The only previously published study on cancer patients was performed before the beginning of the vaccination campaigns, answers remained hypothetical since no vaccine have been approved yet (November 2020) [8], vaccine acceptance was rated at $53.7 \%$. In order to understand patient's perception and better prepare physician's actions towards their patients regarding COVID-19 vaccination, we aimed to measure vaccine acceptability in cancer patients and to investigate determinant factors associated with patient's choice.

\section{Methods}

We conducted a cross-sectional survey with an anonymous self-administered questionnaire delivered to every admitted ambulatory patient in 3 oncology cancer centers in north Tunisia from 1-Feb-2021 to 1-May-2021. Study participants gave informed consent to participate in the survey. Inclusion criteria were all cancer patients, $>18$ years old, regardless of gender, disease type and stage, whether they were under active treatment or follow-up. Participants had to be able to read/understand Arabic and/or French and without impaired cognitive function. The study investigators also shared the web version (in Arabic and French) of the survey link in the most popular social media platform in Tunisia, Facebook. On receiving and clicking the link, participants got auto directed to the informed consent section, followed by the survey questionnaires. From the previous literature review of COVID-19 vaccine hesitancy in the community, it is estimated that about $30 \%$ of the study participants showed hesitancy towards accepting the vaccine. We estimated that a sample size of 323 should give us $80 \%$ power at a confidence level of $95 \%$. The study was stopped when the sample size was reached. It started 6 weeks before the beginning of COVID-19 vaccination in Tunisia and has ended at week 7 since the vaccination initiation in Tunisia.

Questionnaire development Based on a literature review, authors elaborated a bilingual (Arabic, French) questionnaire that was concerning three sections: the first was about patient's demographic features, the second about willingness to take the vaccine, and the third about factors influencing their choice. In the third section, we used a validated survey instrument to measure vaccine acceptance among adults that grouped several independent variables published in 2018 [9]. We adapted several questions to the COVID-19 context (Supp1). Questions from the original questionnaire were translated into Arabic and French using the four-step methodology. Participants had to give their opinion using the 5 -point Likert scale: $1=$ strongly disagree to $5=$ strongly agree. The study was approved by the local ethical committee. In Tunisia, vaccination started on March 19, 2021, the national vaccination platform is called EVAX (evax.tn); it organizes the vaccination process, registration, appointments, and vaccination confirmation. The questionnaire included items about EVAX.

Data analysis Descriptive statistics (frequencies, percentage) were calculated and Ki2 test was conducted for comparisons. Multinomial logistic regression analysis was used to assess odds ratio (OR). OR evaluated the predictive factors of vaccine refusal/hesitancy considering acceptance as a reference. Nominal dependent variable with three categories representing intention to vaccinate: "Yes," "Don't know yet," "No." Independent variables from the questionnaire were analyzed as follows: patients who responded 1 or 2 were grouped as "disagree," patients who responded 3 were considered as "don't know or don't have an opinion" and patients who responded 4 or 5 were grouped as "agree." A two-tailed $p$-value $<0.05$ was considered statistically significant.

\section{Results}

A total of 329 patients participated to the survey. Median age was 54 years old $(\mathrm{SD}=13.4), 78.7 \%(n=259)$ were female. Patients were married in $76.6 \%(n=252), 39.2 \%(n=129)$ had university educational level, $30.1 \%(n=99)$ high school. Most patients $(88.1 \%, n=290)$ were from north cities of Tunisia. Medical history evaluation showed presence of other comorbidities besides cancer in $36.4 \%(n=120)$. Fiftynine percent of patients $(n=194)$ were undergoing chemotherapy and/or radiation therapy. Seventy-one percent of patients were treated in the public sector $(n=234)$. Only $16.1 \%(n=53)$ received the Influenza Vaccine in the last year, $8 \%(n=28)$ had the COVID-19 in the past months. Patients thought that COVID-19 is a severe disease in 78.1\% $(n=257)$ and $14.9 \%(n=49)$ have lost a loved one because of it. Cancer was considered more dangerous than COVID19 by $34.3 \%(n=113)$ of patients, 59\% $(n=194)$ thought that COVID-19 is more severe in cancer patients. Forty-five point six percent of patients $(n=150)$ reported that the pandemic negatively affected their cancer care (Table 1$)$.

Twenty-four percent of patients $(n=79)$ were registered in the Tunisian vaccine platform (EVAX) and $28.2 \%(n=93)$ did not know about it. All patients knew about the vaccine 
Table 1 Patients characteristics according to vaccination choice

\begin{tabular}{|c|c|c|c|c|}
\hline & Yes & No & Don't know yet & $P$ \\
\hline Age $(n=329)$ & $55(\mathrm{SD}=12.9)$ & $48(\mathrm{SD}=13.2)$ & $49(\mathrm{SD}=12.1)$ & 0.0001 \\
\hline $\operatorname{Man}(21.9 \%, n=70)$ & $28.8 \%(n=47)$ & $12.1 \%(n=11)$ & $18.2 \%(n=12)$ & 0.006 \\
\hline University educational level $(39.2 .1 \%, n=129)$ & $46 \%(n=69)$ & $46 \%(n=40)$ & $32.2 \%(n=20)$ & 0.23 \\
\hline Public sector $(71.1 \%, n=234)$ & $96.9 \%(n=114)$ & $75.9 \%(n=66)$ & $88.5 \%(n=54)$ & 0.045 \\
\hline History of comorbidities $(36.4 \%, n=120)$ & $42.9 \%(n=69)$ & $35.5(n=33)$ & $26.8 \%(n=18)$ & 0.12 \\
\hline Treatment ongoing $(58.9 \%, n=194)$ & $62.4 \%(n=98)$ & $56 \%(n=51)$ & $71.4 \%(n=45)$ & \\
\hline History of influenza vaccine in the last year $(16.1 \%, n=53)$ & $21.2 \%(n=35)$ & $14.1 \%,(n=13)$ & $8.3 \%(n=5)$ & 0.053 \\
\hline History of COVID-19 $(8.7 \%, n=28)$ & $7.3 \%(n=12)$ & $11.8 \%(n=11)$ & $7.6 \%(n=5)$ & 0.55 \\
\hline Not registered in EVAX $(47.7 \%, n=157)$ & $68 \%(n=51.1)$ & & & \\
\hline Lost someone close from COVID-19 $(14.8 \%, n=48)$ & $16.9 \%(n=28)$ & $10.8 \%(n=10)$ & $15.2 \%(n=10)$ & 0.73 \\
\hline
\end{tabular}

and $61.4 \%(n=202)$ knew that there are many types of vaccines available. About half of the patients $(53.8 \%, \mathrm{n}=175)$ reported having confidence in the government, and $77.5 \%$ having confidence in their physician.

Among the respondents, 50.5\% $(n=166)$ reported their intent to be vaccinated as soon as the vaccine was available, $28.3 \%(n=93)$ reported to definitely refuse the vaccine, and $21.2 \%(n=70)$ did not make their decision yet. In patients in favor of the vaccination, the reasons of their choice was to protect themselves and their families in $64.1 \%$ $(n=211)$, to protect everyone else in $43.1 \%(n=142)$, and $37.3 \%(n=123)$ would do it to get back to normal life, $35.2 \%$ $(n=116)$ did not give any reason.

In patients who did not support the vaccination, $53.1 \%$ $(n=175)$ did not explain why, 33.1\% $(n=109)$ would not take the vaccine because they fear side effects, $24.9 \%$ ( $n=82$ ) because they do not believe it is effective, and $3 \%$ ( $n=10$ ) because the COVID-19 is not such a severe disease that worth a vaccine.

Seventy-one percent $(n=233)$ of patients agreed that they should keep the freedom to get vaccinated or not, and $31.6 \%$ ( $n=104)$ agreed that it is legitimate for the government to mandate the vaccination.

Patients agreed that the vaccine may affect the efficacy of anti-cancer therapy $(15.5 \%, n=51)$ while $57.4 \%(n=189)$ did not know if it may have this affect. Patients agreed that it may affect the course of the cancer disease in $10.9 \%(n=36)$, and $61.4 \%(n=202)$ did not know if it could.

Patients were asked about their opinion on several items and responded as follows according to the 5-point Likert scale: $39.5 \%$ $(n=130)$ agreed about the idea that the vaccine is not necessary because COVID-19 is not a severe disease. Forty-three percent $(n=144)$ of patients reported that not all vaccines were created equal and that they would prefer some types over others. Nineteen point seven percent $(n=65)$ agreed that the vaccine is not safe, and $40.7 \%(n=134)$ did not know if it is safe or not, $10.5 \%$ $(n=32)$ agreed that the vaccine might change their DNA, and $28.5 \%(n=94)$ agreed that it contains toxic ingredients.
Thirty-three percent ( $n=110$ ) agreed that getting COVID19 will not give a better immunity than that induced by the vaccine and $46.8 \%(n=154)$ did not know what gives better immunity. Thirty-four point eight percent $(n=115)$ agreed that the vaccine will reduce the contagion but $42.2 \%(n=139)$ did not know if it does. Fifty-two percent $(n=172)$ agreed that patients with cancer must have the priority to get the vaccine because it is an at-risk condition.

Twenty percent $(n=68)$ agreed that the vaccine is necessary to end the pandemic.

Thirty-eight percent $(n=127)$ reported preferring to receive the vaccine after the others to ensure its safety.

Multinomial logistic regression was performed to evaluate predictive factors of vaccine resistance in cancer patients as shown in Fig. 1. High educational level, history of comorbidities, history of influenza vaccination, and patient's opinion about the severity of COVID-19 did not predict vaccine resistance. However, patients who think that the vaccine may interfere with treatment efficacy $(\mathrm{OR}=7.28,95 \% \mathrm{CI}$ [2.5-12.32]), or would impact cancer outcome $(\mathrm{OR}=6.14,95 \%$ CI [2.27-16.7]), were significantly more likely to refuse the vaccine. Patients who did not agree that the vaccine is a major weapon against the pandemic $(\mathrm{OR}=6.07,95 \% \mathrm{CI}$ [2.34-9.52]) and that it could reduce the virus transmission $(\mathrm{OR}=7.34,95 \% \mathrm{CI}$ [4.22-11.81]), and were also significantly more likely to reject the vaccination. Safety concerns were also predictive factors (Fig. 1). Confidence level in the authorities played a significant role is patients' acceptance of the vaccine; indeed, patients not registered $(\mathrm{OR}=5.9,95 \% \mathrm{CI}$ [1.58-8.7]) or not informed about the national vaccination platform EVAX $(\mathrm{OR}=5.51,95 \% \mathrm{CI}$ [2.1-7.9]) were more likely to be against the vaccine.

\section{Discussion}

The present study showed that COVID-19 vaccine acceptance in cancer patients was $50.5 \%$, with a relatively high proportion of resistant patients $(28.3 \%)$. Concerns about 


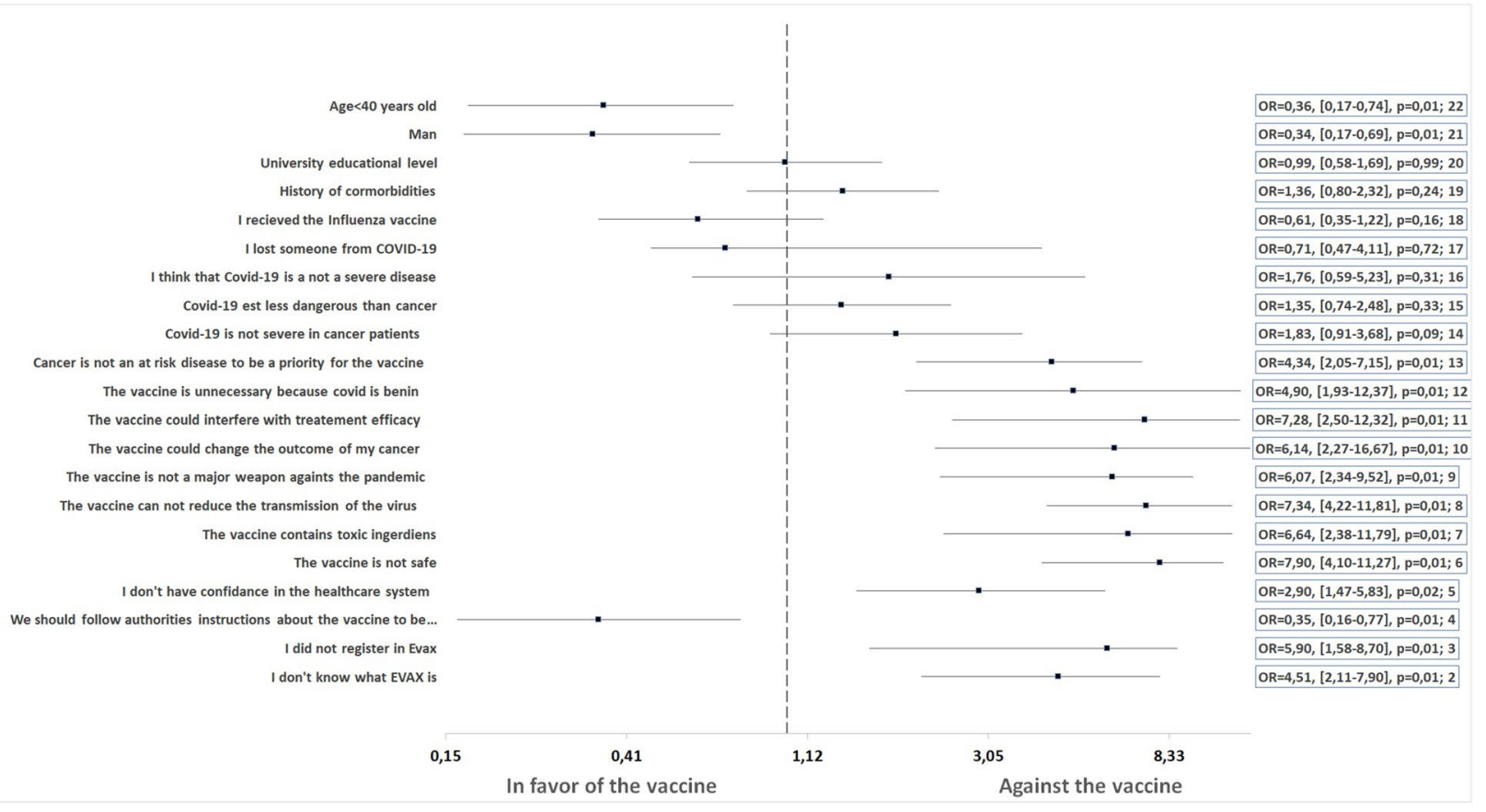

Fig. 1 Odds ratio of factors associated with vaccine choice

cancer outcome and vaccine safety were significant predictive factors of vaccine resistance. Patients who did not agree that the vaccine may help fight the pandemic/ reduce the virus transmission and those with poor confidence in the healthcare system were also more likely to not support the vaccine. At the time of writing this paper, vaccination process is ongoing at different speeds around the world; some countries are very advanced, others are struggling [10]. One of the major challenges is vaccine acceptance especially with the important amount of misinformation on the Internet [11]. Cancer patients suffered from the pandemic that impacted several aspects of their disease management. When it comes to vaccine, patients and oncologists face a dilemma of prioritizing COVID19 vaccination while vaccine supply is limited [5]. In a previous study, vaccine acceptance among French cancer patients was $53.7 \%$ [8]; we observed the same rate among Tunisian patients. These results could be considered lower than studies reporting vaccine acceptance among the general population ranging from 67 to $82 \%$ [12-15]. Several vaccine resistance factors reported in our study are already known from previous studies in the general population, such as age and gender [8], trust in government decisions [12], safety concerns. Conversely, we did not observe an impact of educational level [16], influenza vaccine history [8, 17], knowing people who died from COVID-19 [18], and perceiving COVID-19 as a threat to self [19].

In our population, patients who think that the vaccine may interact with the chemotherapy or impact cancer course were more likely to reject the vaccine. Furthermore, $48 \%$ of patients did not agree that cancer is an at-risk condition that gives patients priority of the vaccine. Oncologists need to communicate with their patients about these specific points; they should have an active attitude to educate patients during outpatient and inpatient consultations. Another new finding that came out from the present study was what patients think about the vaccine role in fighting the pandemic and in stopping virus transmission. Education remains a crucial way to "correct" these perceptions. Patients need trusted sources of information. In our population, $46.2 \%$ of patients did not have confidence in the government, and $77.5 \%$ had confidence in their physician. This leaves doctors with a big responsibility to give complete and clear information to their patients. Indeed, in Tunisia, health care authorities launched an online platform for vaccine registration www. evax.tn on February 5, 2021. When first launched, cancer patients were not considered among the priority population like those with diabetes, renal, or liver failure, age $>75$ years old, and health care workers. The Tunisian society of medical oncology implemented efforts in order to include cancer patient's along with other priority populations and obtained authorities approval $[20,21]$. Governments must be ready to ensure large-scale, equitable access, and distribution of a COVID-19 vaccine, but also should work on communication strategies to gain more population confidence [22].

Some limitations of the present study could be highlighted: this is a cross-sectional study giving only a picture at the moment of the survey, but it is the only published 
study among cancer patients after the approval and initiation of the vaccination campaign. Male patients were underrepresented; this is a selection bias since breast cancer is the predominant malignancy in all participating centers. Authors used a self-administered questionnaire requiring reading skills, illiterate population was not included, and we expect a different acceptance rate among them; it is highly probable that specific actions need to be initiated in this particular population.

In conclusion, the present study showed that 1 over two cancer patients was spontaneously willing to take COVID19 vaccine, but there is a room for improvement toward decreasing vaccine hesitancy. Oncologists should focus on communicating on two major points with their patients: the impact of the vaccine on cancer/cancer treatment the role of the vaccine in fighting COVID-19.

Supplementary Information The online version contains supplementary material available at https://doi.org/10.1007/s00520-021-06419-y.

Author contribution Study idea and design: Nesrine Mejri, Emna Ouertani; data collection: Nesrine Mejri, Emna Ouertani, Mariem Bohli; data analysis: Mejri Nesrine, Yosra Berrazegua; writing: Nesrine Mejri,Yosra Berrazega, Haifa Rachdi; review: Boussen Hamouda, Lotfi Kochbti.

Data availability Data is available on request.

Code availability Software application available.

\section{Declarations}

Ethics approval Approved by the local ethical committee.

Consent to participate Obtained.

Consent for publication Obtained.

Conflicts of interest The authors declare that they have no conflict of interest.

\section{References}

1. Al-Quteimat OM, Amer AM (2020) The impact of the COVID-19 pandemic on cancer patients. Am J Clin Oncol. https://doi.org/10. 1097/COC.0000000000000712

2. Liang W, Guan W, Chen R et al (2020) Cancer patients in SARSCoV-2 infection: a nationwide analysis in China. Lancet Oncol 21(3):335-337

3. Lee LYW, Cazier J-B, Starkey T et al (2020) COVID-19 prevalence and mortality in patients with cancer and the effect of primary tumour subtype and patient demographics: a prospective cohort study. Lancet Oncol 21(10):1309-1316
4. Nile SH, Nile A, Jalde S, Kai G (2021) Recent advances in potential drug therapies combating COVID-19 and related coronaviruses-a perspective. Food Chem Toxicol 154:112333

5. Ribas A, Sengupta R, Locke T et al (2021) Priority COVID-19 Vaccination for Patients with Cancer while Vaccine Supply Is limited. Cancer Discov 11(2):233-236

6. Dooling K, McClung N, Chamberland M et al (2020) The advisory committee on immunization practices' interim recommendation for allocating initial supplies of COVID-19 vaccine - United States, 2020. MMWR Morb Mortal Wkly Rep 69(49):1857-1859

7. de Figueiredo A, Simas C, Karafillakis E et al (2020) Mapping global trends in vaccine confidence and investigating barriers to vaccine uptake: a large-scale retrospective temporal modelling study. Lancet 396(10255):898-908

8. Barrière J, Gal J, Hoch B et al (2021) Acceptance of SARS-CoV-2 vaccination among French patients with cancer: a cross-sectional survey. Ann Oncol. https://doi.org/10.1016/j.annonc.2021.01.066

9. Sarathchandra D, Navin MC, Largent MA, McCright AM (2018) A survey instrument for measuring vaccine acceptance. Prev Med 109:1-7

10. Press VG, Huisingh-Scheetz M, Arora VM (2021) Inequities in technology contribute to disparities in COVID-19 vaccine distribution. JAMA Health Forum 2(3):e210264

11. Latkin CA, Dayton L, Miller JR et al (2021) Behavioral and attitudinal correlates of trusted sources of COVID-19 vaccine Information in the US. Behav Sci 11(4):56

12. Lazarus JV, Ratzan SC, Palayew A et al (2020) A global survey of potential acceptance of a COVID-19 vaccine. Nat Med. https:// doi.org/10.1038/s41591-020-1124-9

13. Malik AA, McFadden SM, Elharake J, Omer SB (2020) Determinants of COVID-19 vaccine acceptance in the US. EClinicalMedicine 26:100495

14. Kreps S, Prasad S, Brownstein JS et al (2020) Factors Associated With US adults' likelihood of accepting COVID-19 vaccination. JAMA Netw Open 3(10):e2025594-e2025594

15. Robertson E, Reeve KS, Niedzwiedz CL et al (2021) Predictors of COVID-19 vaccine hesitancy in the UK household longitudinal study. Brain Behav Immun 94:41-50

16. El-Elimat T, AbuAlSamen MM, Almomani BA et al (2021) Acceptance and attitudes toward COVID-19 vaccines: a crosssectional study from Jordan. PLOS ONE 16(4):e0250555

17. Alqudeimat Y, Alenezi D, AlHajri B et al (2021) Acceptance of a COVID-19 vaccine and its related determinants among the general adult population in Kuwait. MPP. https://doi.org/10.1159/00051 4636

18. Yurttas B, Poyraz BC, Sut N et al (2021) Willingness to get the COVID-19 vaccine among patients with rheumatic diseases, healthcare workers and general population in Turkey: a web-based survey. Rheumatol Int 41(6): 1105-1114

19. Vallée A, Fourn E, Majerholc C et al (2021) COVID-19 Vaccine hesitancy among French people living with HIV. Vaccines 9(4):302

20. STOM Archives. [http://kapitalis.com/tunisie/tag/stom/]

21. (2021) Vaccin anti-covid-19: La STOM appelle à intégrer les malades du cancer sous chimiothérapie dans la liste des catégories prioritaires. La Presse de Tunisie

22. Salwa J, Robertson C (2021) Designing an independent public health agency. N Engl J Med 384(18):1684-1687

Publisher's note Springer Nature remains neutral with regard to jurisdictional claims in published maps and institutional affiliations. 